\title{
GROUPS OF DIMENSION 1 ARE LOCALLY FREE
}

\author{
BY JOHN STALLINGS
}

Communicated by S. Smale, November 15, 1967

Our result is slightly more general than

ThEOREM 1. A torsion-free, finitely presented group $G$, with infinitely many ends, can be written as a nontrivial free product $G_{1} * G_{2}$.

The condition "finitely presented" can be weakened to: There is a finite complex $K$ and a regular covering space $\widetilde{K}$ with $H^{1}(\widetilde{K})=0$, such that $G$ is isomorphic to the group of covering translations of $\tilde{K}$.

From this we deduce

THEOREM 2. If a finitely generated group $G$ has cohomological dimension $\leqq 1$, then $G$ is free [1].

This is another way of stating the title theorem. Another consequence is

THEOREM 3. If a finitely generated group $G$ has a free subgroup of finite index, and if $G$ is torsion-free, then $G$ is free [3].

(The references are to papers where these results have been conjectured.)

We shall indicate briefly how to prove Theorems 1 and 2. Details will appear elsewhere.

We use cohomology with coefficient group $\boldsymbol{Z}_{2}$. Ordinary cohomology is called $H^{n}(X)$. Cohomology with finite cochains is $H_{f}^{n}(X)$. By $Z_{2} G$ we denote the group ring of $G$ with coefficient ring $Z_{2}$; modules, projective modules, etc., are with reference to this ring; if $M$ is a module, $M^{\star}$ means $\operatorname{Hom}_{Z_{2} G}\left(M, Z_{2} G\right)$.

To say that a group $G$ has infinitely many ends, means that $H^{1}\left(G ; \boldsymbol{Z}_{2} G\right)$ is more than $\boldsymbol{Z}_{2}$. In terms of the regular covering space $\tilde{K}$, on which $G$ acts freely with quotient complex $K$, where $H^{1}(\tilde{K})=0$, this means that $H_{f}^{1}(\widetilde{K})$ contains more than two elements.

We suppose that $K$ is a finite simplicial complex with ordered vertices; on this and on $\tilde{K}$ we have the standard cup-product of cochains defined, denoted by $\cdot$.

By a minimal 1-cocycle $P$ we mean a finite 1-cocycle on $\tilde{K}$, which is nonzero in $H_{f}^{1}(\tilde{K})$, and which is, among all such, one involving the fewest 1 -simplexes. 
Since $H^{1}(\tilde{K})=0, P$ cobounds two infinite 0 -cochains $E$ and $E^{*}$.

LEMMA 1. E and $E^{*}$ are connected.

This means, in the case of $E$, say, that any two 0 -simplexes in $E$ can be joined by a finite chain of 1-simplexes, all of whose end-points lie in $E$. The reason for this is that otherwise $P$ would be a disjoint sum of two cocycles, both non-trivial; one of these would be nonzero in $H_{f}^{1}(K)$ and smaller than $P$.

The group $G$ acts on cocycles and cochains. If $E$ and $F$ are 0 cochains, then $E \cdot F$, the cup-product, is their intersection.

LEMMA 2. If all these cochains are nonzero:

$$
E \bullet g E, E \bullet g E^{*}, E^{*} \cdot g E, E^{*} \cdot g E^{*}
$$

then $P$ and $g P$ have some 1-simplex in common.

For, we use Lemma 1 and the impossibility of writing $P$ as a sum of disjoint cocycles to show that $P \cdot g P \neq 0$. If the simplicial cup product of two simplicial 1-cocycles is nonzero, they actually in tersect.

Lemma 3. One, at least, of the 0-cochains in Lemma 2 is finite. $P$.

For, one of them has coboundary involving fewer 1-simplexes than

LEMma 4. If $g$ has infinite order, and if $P$ and $g P$ represent the same element in $H_{f}^{1}(\widetilde{K})$, then $H_{f}^{1}(\widetilde{K})=Z_{2}$; i.e., $G$ has two ends.

Roughly speaking, $\tilde{K}$ is made up of a doubly infinite telescope whose sections are $g^{n} F$, where $F$ is a finite 0 -cochain with coboundary $P-g P$.

Lemma 5. If $G$ has infinitely many ends and is torsion-free, and $1 \neq g \in G$, then exactly one of the following is finite: $E \cdot g E, E \cdot g E^{*}$, $E^{*} \cdot g E, E^{*} \cdot g E^{*}$.

For, by Lemma 3, at least one is finite. If two were finite, then $P$ or $P-g P$ or $g P$ would represent 0 in $H_{f}^{1}(\tilde{K})$, contradicting Lemma 4 .

Thus, $G-\{1\}$ splits into four sets, denoted respectively

$$
A A, A A^{*}, A^{*} A, A^{*} A^{*} \text {. }
$$

Formally, let $X, Y, Z$ stand for $A$ or $A^{*}$, and $\left(A^{*}\right)^{*}=A$.

LEMMA 6. (a) $(X Y)^{-1}=Y X$.

(b) $X Y \cdot Y^{*} Z \subset X Z$. 
(c) For every $g \in G$ there is an upper bound to the numbers $n$, for which there are $X_{0}, X_{1}, \cdots, X_{n}$, and $g_{i} \in X_{i-1} X_{i}^{*}$, such that $g=g_{1} g_{2} \cdots g_{n}$. (d) None of the sets $X Y$ is empty.

The proof of this is mostly computational.

An irreducible element $g \in X Y$ is one that cannot be written as $g_{1} g_{2}$ for $g_{1} \in X Z, g_{2} \in Z^{*} Y$.

Let $A$ denote $\{1\}$ together with all irreducible elements of $A A$. Let $B$ denote $\{1\}$ together with all irreducible elements of $A^{*} A^{*}$. These are subgroups of $G$.

Lemma 7. (a) If there is no irreducible element of $A A^{*}$, then $G$ is the free product of $A$ and $B$.

(b) If there is an irreducible element $\gamma \in A A^{*}$, then $G$ is the free product of $A$ and the infinite cyclic group generated by $\gamma$.

This is a combinatorial consequence of Lemma 6. It implies Theorem 1.

As for Theorem 2, it is not hard to prove that such a $G$ can be represented as the group of covering translations of $\tilde{K}$ over a finite complex $K$, with $H^{1}(\tilde{K})=0$. Also, such a $G$ is torsion-free.

LEMMA 8. A torsion-free group with two ends is infinite cyclic.

This is a theorem of C. T. C. Wall [4, Lemma 4.1]. Using this and Grushko's Theorem [2], we can prove Theorem 2 by induction on the number of generators of $G$, using Theorem 1 to split $G$ into a free product, each factor of which has fewer generators. And so we need to prove:

LEMMA 9. If $G$ is a nontrivial, finitely generated group of cohomological dimension 1 , then $H^{1}\left(G ; Z_{2} G\right) \neq 0$.

Since $G$ is 1-dimensional and finitely generated, the kernel $M$ of the augmentation $\boldsymbol{Z}_{2} G \rightarrow \boldsymbol{Z}_{2}$ is a finitely generated projective module. The cohomology of $G$ with coefficient group $Z_{2} G$ now fits into an exact sequence:

$$
0 \rightarrow H^{0}\left(G ; Z_{2} G\right) \rightarrow\left(Z_{2} G\right)^{\star} \rightarrow(M)^{\star} \rightarrow H^{1}\left(G ; Z_{2} G\right) \rightarrow 0
$$

Since $G$ is finite dimensional, it has no elements of finite order. Hence $G$ is infinite; this implies $H^{0}\left(G ; \boldsymbol{Z}_{2} G\right)=0$. If, additionally, $H^{1}\left(G ; \boldsymbol{Z}_{2} G\right)$ were 0 , the exact sequence would say that $\left(Z_{2} G\right)^{\star} \rightarrow M^{\star}$ is an isomorphism. Since these are finitely generated projective modules, the original map $M \rightarrow Z_{2} G$ would have to be an isomorphism, contrary to the fact that it has cokernel $\boldsymbol{Z}_{2}$. 
This derivation of Theorem 2 from Theorem 1 was shown to us by C. T. C. Wall.

\section{REFERENCES}

1. S. Eilenberg and T. Ganea, On the Lusternik-Schnirelmann category of abstract groups, Ann. of Math. 65 (1957), 517-518.

2. A. G. Kurosh, The theory of groups, Transl. by K. A. Hirsch, Chelsea, New York, 1956.

3. J.-P. Serre, Sur la dimension cohomologique des groupes profinis, Topology 3 (1965), 413-420.

4. C. T. C. Wall, Poincare complexes. I, Ann. of Math. 86 (1967), 213-245.

University of California, Berkeley

\section{ON THE NORM OF STABLE MEASURES ${ }^{1}$}

BY LUDWIG ARNOLD AND JOHANNES MICHALICEK

Communicated by R. Creighton Buck, October 12, 1967

1. Limits of convolution powers and stable measures. Let $M(R)$ denote the Banach algebra of all complex-valued regular finite measures defined on the Borel sets of the real line $R$, where multiplication is defined by convolution, and

$$
\|\mu\|=\sup \sum\left|\mu\left(R_{i}\right)\right|,
$$

the supremum being taken over all finite collections of pairwise disjoint sets $R_{i}$ whose union is $R$. Let $B(R)$ be the set of all Fourier transforms of measures in $M(R)$.

In [1], we characterized all possible limits

$$
\lim _{n \rightarrow \infty}\left(\hat{\nu}\left(t / B_{n}\right)\right)^{n} \exp \left(i t A_{n}\right)=\hat{\mu}(t) \text { for all } t \neq 0,
$$

where $A_{n} \in R, B_{n}>0, \hat{\nu}, \hat{\mu} \in B(R)$. This is a generalization of an old problem in probability theory (see e.g. [4]). One can show that a measure $\mu$ appears as a limit if and only if it is stable, i.e. has the following property: For all $a>0, b>0$ there exist $c>0$ and $\gamma \in R$ such that

$$
\hat{\mu}(a t) \hat{\mu}(b t)=\hat{\mu}(c t) \exp (i \gamma t) \quad \text { for all } t \in R \text {. }
$$

${ }^{1}$ Sponsored by the Mathematics Research Center, United States Army, Madison, Wisconsin, under Contract No. DA-31-124-ARO-D-462. 